\title{
Antenatal nutritional supplementation and autism spectrum disorders in the Stockholm youth cohort: population based cohort study
}

\author{
Elizabeth A DeVilbiss, ${ }^{1}$ Cecilia Magnusson, ${ }^{2}$ Renee M Gardner, ${ }^{2}$ Dheeraj Rai, ${ }^{3}$ \\ Craig J Newschaffer, ${ }^{1,4}$ Kristen Lyall, ${ }^{4}$ Christina Dalman, ${ }^{2}$ Brian K Lee ${ }^{1}$
}

\begin{abstract}
${ }^{1}$ Department of Epidemiology and Biostatistics, Dornsife School of Public Health, Drexel University, 3215 Market St, Philadelphia, PA, 19104, USA

${ }^{2}$ Public Health Epidemiology, Department of Public Health

Sciences, Karolinska Institutet,

Stockholm, Sweden

${ }^{3}$ Centre for Academic Mental Health, School of Social and

Community Medicine, University

of Bristol, Bristol, UK

${ }^{4}$ AJ Drexel Autism Institute,

Philadelphia, PA, USA

Correspondence to:

E A DeVilbiss

ead77@drexel.edu

Additional material is published online only. To view please visit the journal online.

Cite this as: $B M J$ 2017;359:j4273 http://dx.doi.org/10.1136/bmj.j4273
\end{abstract}

Accepted: 3 September 2017

\section{ABSTRACT}

OBJECTIVE

To determine whether nutritional supplementation during pregnancy is associated with a reduced risk of autism spectrum disorder (ASD) with and without intellectual disability in offspring.

\section{DESIGN}

Observational prospective cohort study using multivariable logistic regression, sibling controls, and propensity score matching.

SETTING

Stockholm County, Sweden.

\section{PARTICIPANTS}

273107 mother-child pairs identified through population registers. The study sample was restricted to children who were aged 4 to 15 years by the end of follow-up on 31 December 2011 and were born between 1996 and 2007.

\section{EXPOSURES}

Multivitamin, iron, and folic acid supplement use was reported at the first antenatal visit.

\section{MAIN OUTCOME MEASURE}

Diagnosis of ASD with and without intellectual disability in children determined from register data up to 31 December 2011.

\section{RESULTS}

Prevalence of ASD with intellectual disability was $0.26 \%$ (158 cases in 61934$)$ in the maternal multivitamin use group and $0.48 \%$ ( 430 cases in 90480 ) in the no nutritional supplementation use group. Maternal multivitamin use with or without additional iron or folic acid, or both was associated with lower odds of ASD with intellectual disability in the child compared with mothers who did not use multivitamins, iron, and folic acid (odds ratio

\section{WHAT IS ALREADY KNOWN ON THIS TOPIC}

Evidence from observational studies is inconsistent about whether maternal supplementation with multivitamins, iron, or folic acid is protective against autism spectrum disorder (ASD) in offspring

Although the cause of autism spectrum disorder may differ by presence of intellectual disability, few studies have examined relations between nutritional supplements and ASD based on level of cognitive function

\section{WHAT THIS STUDY ADDS}

This population based study of a large cohort in Stockholm supported a possible inverse association between maternal use of multivitamin supplements in early pregnancy and ASD with intellectual disability in offspring compared with no

maternal use of multivitamins, iron, or folic acid

$0.69,95 \%$ confidence interval 0.57 to 0.84 ). Similar estimates were found in propensity score matched $(0.68,0.54$ to 0.86$)$ and sibling control $(0.77,0.52$ to 1.15$)$ matched analyses, though the confidence interval for the latter association included 1.0 and was therefore not statistically significant. There was no consistent evidence that either iron or folic acid use were inversely associated with ASD prevalence.

\section{CONCLUSIONS}

Maternal multivitamin supplementation during pregnancy may be inversely associated with ASD with intellectual disability in offspring. Further scrutiny of maternal nutrition and its role in the cause of autism is recommended.

\section{Introduction}

With the heritability of autism spectrum disorders (ASD) estimated to be between $50 \%$ and $80 \%,{ }^{1}{ }^{2}$ non-heritable risk factors contribute to a substantial proportion of ASD risk. Research suggests that ASD develops antenatally. ${ }^{3-5}$ Maternal nutrition influences neurodevelopment ${ }^{6-8}$ and could influence the risk of ASD. ${ }^{9}$ However, results from population based studies have been inconsistent. An American casecontrol study (CHildhood Autism Risks from Genetics and Environment (CHARGE) $)^{10}$ and a Norwegian cohort study (Norwegian Mother and Child Cohort Study $(\mathrm{MoBa}))^{11}$ reported reduced risks of ASD with maternal folic acid supplementation and intake in the periconceptional period and during early pregnancy. Conversely, a study of the Danish National Birth Cohort (DNBC) reported no associations between early folic acid or multivitamin supplementation and risk of ASD. ${ }^{12}$ Maternal iron intake ${ }^{13}$ and antenatal vitamin use $\mathrm{e}^{14}$ were both associated with a reduced risk of ASD in CHARGE, whereas no association between ordinary multivitamin use and ASD was reported. ${ }^{14}$ Clarifying the potential role of nutritional supplements is difficult, given the potential for the cause of ASD to differ by the presence of intellectual disability, ${ }^{15-17}$ although this has not been well studied in the context of nutritional supplementation.

This association might be confounded by maternal characteristics $^{9}{ }^{18}$-namely, mothers who have a given dietary pattern are likely to be different from those who do not have that dietary pattern; potential differences may include socioeconomic characteristics, disease status, healthy behaviours, or pregnancy characteristics. ${ }^{9}{ }^{18}$ If these differences are not properly accounted for, then results may be owing to confounding. 
In the present study, we used three analytic strategies to elucidate potential associations between maternal nutritional supplementation with multivitamins, iron, and folic acid and risk of ASD in offspring with and without intellectual disability. These included sibling controls to account for familial, environmental, and genetic confounding; propensity score matching to ensure balanced comparisons between exposed and unexposed children; and multivariable regression with adjustment for a large number of covariates. The sibling matched analyses serve as a guide as to whether estimates are confounded by unobserved shared familial confounders but have low statistical power. The propensity score matched analyses are intended to help determine whether confounding from observed characteristics unduly influences results but are not necessarily generalizable. The multivariable regression analysis in the whole sample provides the most generalizable and interpretable estimates but is the most susceptible to limitations such as confounding from observed and unobserved characteristics. The study was designed to use the different strengths of the three analytic approaches to gauge robustness of findings.

\section{Methods}

\section{Study sample}

Data were drawn from the Stockholm youth cohort, a population register based cohort of children living in Stockholm County, Sweden, for at least four years between 2001 and 2011. ${ }^{19}$ The study sample was, for the purpose of availability of data on supplement use, restricted to children who were born in Sweden between 1996 and 2007 (ie, aged 4 to 15 years at the end of follow-up on 31 December 2011). We excluded children who were not in the medical birth register $(n=17029)$, not linked to birth mother $(n=41)$, adopted $(\mathrm{n}=193)$, or had missing data on family disposable income $(n=298)$ or maternal age $(n=5)$ (fig 1). Children are recorded in the medical birth register if birth records are sent from the delivery hospital to the register. ${ }^{20}$ An earlier report about the quality of the medical birth register ${ }^{20}$ cited that up to $2 \%$ of children born in Sweden are not recorded in the register; however, the report acknowledged that this rate has recently increased.

Sibling analyses were based on a subset of cohort, with singletons removed; propensity score analyses were based on a subset of matched groups; and multivariable regression analyses were based on the whole cohort (fig 1). Siblings were restricted to members of the study sample born within the study period and were defined as having the same birth mother.

\section{Case ascertainment}

Data on autism spectrum disorder (ASD) were drawn from computerized registers covering all pathways of ASD diagnosis and care in Stockholm County. Case ascertainment is described in detail elsewhere. ${ }^{19}$ Paediatric healthcare and habilitation (ie, disability support) services are available free of charge in Sweden.

Developmental screening occurs at regular intervals, whereby children with suspected autism are referred for structured diagnostic assessments by specialists. ${ }^{21}$ ASD is recorded using ICD-10 (international classification of diseases, 10th revision) code F84 and DSM-IV (Diagnostic and Statistical Manual of Mental Disorders, fourth edition, text revision) code 299 in registers covering child and adolescent mental health, habilitation, and paediatric inpatient and outpatient services. In this study, outcomes of interest were any ASD and ASD with or without a recorded intellectual disability in registers using ICD-10 codes F70-79 and DSM-IV codes $317-319^{16}$ and supplemented with data from the habilitation register, which categorizes autism by comorbid intellectual disability. ${ }^{19}$ For any ASD, a review of medical records indicated that $96.0 \%$ of

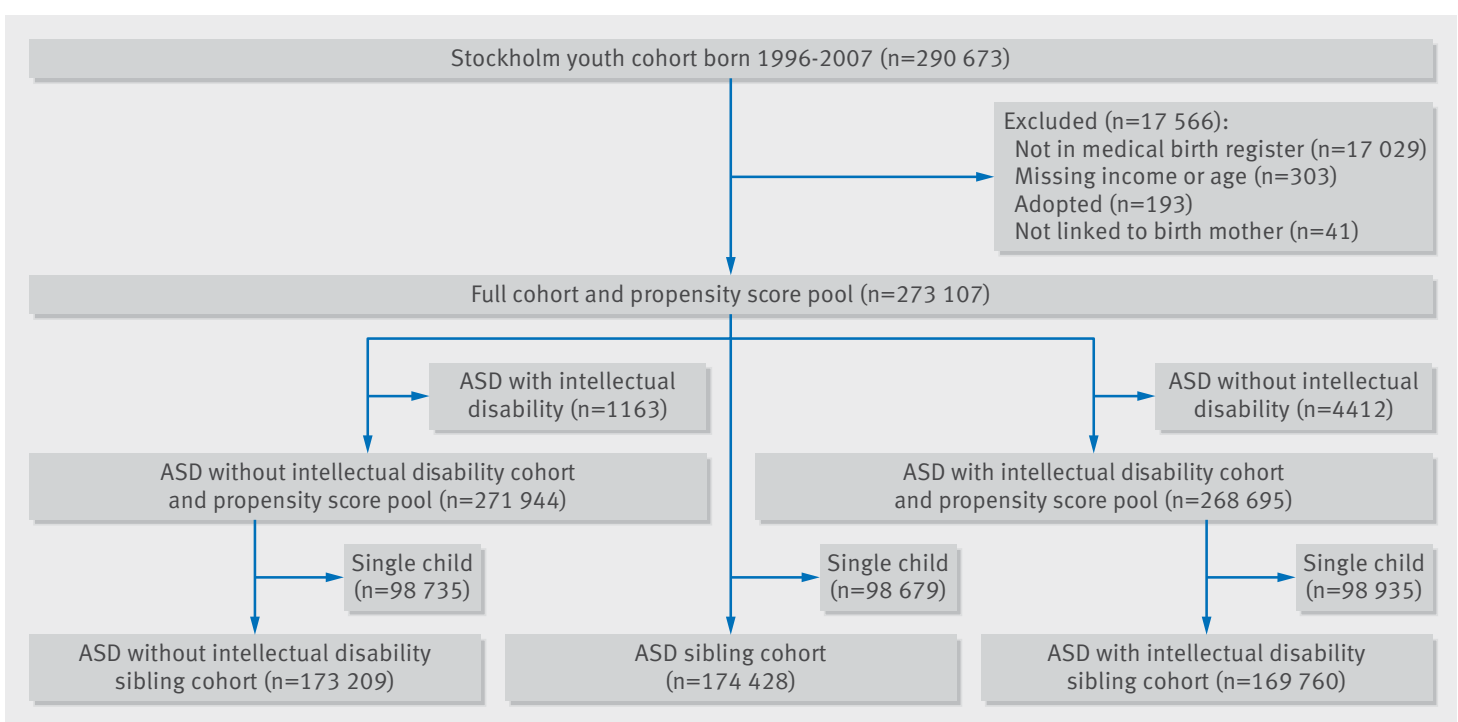

Fig 1 | Derivation of study samples. ASD=autism spectrum disorder 
recorded cases were consistent with diagnostic criteria. When assessing the validity of ASD case status by cooccurring intellectual disability, ASD with intellectual disability was confirmed in 68 of 90 cases (76\%); 17 of the 22 cases of non-confirmed ASD with intellectual disability (77\%) had ASD but no documentation of intellectual disability. ${ }^{19}$

\section{Maternal supplement use}

Web appendix 1 provides recommendations about supplement use for pregnant women during the study period. Briefly, pregnant women are advised to take $400 \mu \mathrm{g} /$ day of folic acid during the first trimester. ${ }^{22}$ Through 2008, $100 \mathrm{mg} /$ day of iron was recommended for pregnant women during weeks 20 to 24 but is now only recommended ( $200 \mathrm{mg} /$ day) for pregnant women who are anemic. ${ }^{23}$ No guidelines were available for multivitamin use during the study period. Self reported supplement and drug use was assessed at the first antenatal visit. Gestational week at the first antenatal visit was recorded in the medical birth register from mid-1995 onwards (median 10.7 weeks, interquartile range $9.0-12.7$ weeks). ${ }^{20}$ Most women attended scheduled antenatal care visits; these consultations are free of charge in Sweden. ${ }^{24}$ Using a standardized form, a midwife interviewed the woman at the first visit and asked about any drug use during pregnancy. Drug information was recorded by the midwife and later transcribed into the medical birth register as Anatomical Therapeutic Chemical (ATC) classification codes (see web appendix eTable 2) or free text.

We individually created variables based on ATC code and free text matches (see web appendix 1), and combined these to create five non-overlapping usage groups: multivitamins, iron, iron and folic acid, folic acid, or none (no use of multivitamins, iron, or folic acid). For all analyses, this last group served as the reference category against which we compared other supplement use groups. The multivitamins category included those women who noted use of any multivitamin, regardless of any combined use with additional iron or folic acid, or both. Multivitamin formulations in Sweden commonly include multiminerals; we included these combinations in the multivitamins category. Multiminerals such as calcium or magnesium supplements without evidence of inclusion of iron or folic acid in the formulation were classified as none. Since medical birth register data do not reliably discern between multivitamins and antenatal vitamins, they were combined and are referred to as multivitamins. Register data also do not contain information about dosage, timing of initiation, frequency, or duration. Midwife interview of self reported maternal folic acid use has been reported to correspond well with plasma folate levels among Swedish women, ${ }^{25}$ so it is not unreasonable that other supplements may also correspond well.

\section{Covariates}

National identification numbers enabled record linkage between national registers held by Statistics Sweden and the National Board of Health and Welfare.
Registers utilised include the medical birth register for child and pregnancy characteristics, ${ }^{2026}$ the integrated database for labour market research for socioeconomic factors, ${ }^{15}$ and national and regional health registers for inpatient, outpatient, prescription drug, and psychiatric data. ${ }^{19}$ All covariates were chosen based on published relations with $\mathrm{ASD}^{27}$ or the use of supplements, or both.

Child factors were sex, birth year (unordered single year categories), and years of residence in Stockholm County (continuous) to account for length of followup. Sociodemographic data included maternal country of birth (Sweden, other European, non-European), maternal education ${ }^{28}$ (pre-secondary, secondary, post-secondary, missing), and disposable family income (fifths). Family income was calculated after tax deductions, adjusted for family size, and based on birth year to account for inflation. Maternal covariates included age $(<25,25-29,30-34, \geq 35$ years), parity $(1,2, \geq 3)$, smoking at first antenatal visit (yes, no, missing), body mass index (BMI) at first antenatal visit (underweight: <18.5, normal: 18.5-24.9, overweight: 25-29.9, obese: $\geq 30 \mathrm{~kg} / \mathrm{m}^{2}$, missing). Web appendix 1 describes how we managed missing education, smoking, and BMI data.

Lifetime history of maternal neurologic or psychiatric conditions before the child's birth (anxiety disorders, ASD, bipolar disorder, depression, intellectual disability, non-affective psychoses, stress related disorders, and epilepsy: yes or no for each (see supplemental eTable 3)) were ascertained from the national patient register and the Stockholm County adult outpatient psychiatric register. Both registers have high validity. ${ }^{29}$

Medication use (antiepileptics and antidepressants: yes or no for each) was ascertained using the medical birth register (self reported at first antenatal visit by interview, available for the entire study period) and the prescription drug register (records of prescribed dispensed drugs, available for births from 2006 to 2007). Some validation work has been conducted examining the concordance of maternal drug use during pregnancy in the prescription drug register and the medical birth register. For example, $69 \%$ of mothers with antiepileptic use recorded in the prescription drug register also had corresponding information in the medical birth register. ${ }^{30}$

\section{Primary analyses}

Odds ratios with 95\% confidence intervals between supplement intake and each outcome (ASD with intellectual disability, ASD without intellectual disability, and any ASD) were calculated using SAS software, version 9.3 (SAS Institute, Cary, NC) and R.

We carried out sibling matched analyses using conditional logistic models stratified by mother and adjusted for sex, parity, and birth year.

Propensity score analyses were conducted separately for each category of supplement use. Propensity scores were estimated using logistic regression including all covariates with observed or hypothesised associations 
with supplementation or ASD or both. Odds ratios were calculated for matched samples using generalised estimating equation models with logit link, clustered by mother. Details of propensity score matching are described in web appendix 1.

Multivariable regression analyses used generalised estimating equation models with logit link, clustered by mother. Base models were adjusted for child sex and birth year. Fully adjusted models included all covariates mentioned.

\section{Sensitivity analyses}

Multivitamin categorization

As the multivitamin group contains all mothers that have taken any multivitamins, we performed a post hoc sensitivity analysis including mothers who used multivitamins without additional iron or folic acid to isolate the association between ASD with intellectual disability and multivitamins on their own as opposed to a combination. Since primary analyses were conducted using mutually exclusive exposure groups, we also analysed an alternate approach using indicator variables for supplement use to evaluate the specific associations of each supplement while adjusting for the other supplements.

\section{Maternal hospital stays}

We hypothesised that underlying, possibly unobserved, health factors might influence an apparent increase in risk associated with use of only folic acid. To investigate, we carried out a post hoc sensitivity analysis, stratifying by number of maternal hospital stays during pregnancy excluding the delivery (0 $v \geq 1$, in approximately a 45 to 55 split). Although we considered other health factors as stratification factors (eg, maternal epilepsy), these analyses were not possible owing to the small number of women who used folic acid and had children with ASD with $(\mathrm{n}=15)$ or without $(\mathrm{n}=63)$ intellectual disability.

\section{Unobserved confounding}

We conducted a sensitivity analysis to determine how robust observed associations of multivitamin use with ASD with intellectual disability were to the presence of a hypothetical, unobserved confounder (see web appendix 1). ${ }^{31}$

\section{Patient involvement}

No patients were involved in setting the research question or the outcome measures, nor were they involved in developing plans for design or implementation of the study. No patients were asked to advise on interpretation or writing up of results. There are no plans to disseminate the results of the research to study participants or the relevant patient community.

\section{Results}

\section{Study sample}

The study sample consisted of 273107 motherchild pairs, categorized into groups: multivitamins $(\mathrm{n}=62840,23.0 \%)$, iron only $(\mathrm{n}=90138,33.0 \%)$, iron and folic acid $(n=25445,9.3 \%)$, folic acid only $(\mathrm{n}=2789,1.0 \%)$, and none of the above $(\mathrm{n}=91895$, $33.7 \%$ ) (table 1). The multivitamin group included multivitamin only $(\mathrm{n}=17539,6.4 \%)$, multivitamin and iron $(n=37046,13.6 \%)$, multivitamin and folic acid $(\mathrm{n}=1602,0.6 \%)$, and multivitamin, iron, and folic acid $(\mathrm{n}=6653,2.4 \%)$.

For the groups, event rates (ASD, ASD with intellectual disability, ASD without intellectual disability) were, respectively: multivitamins (1.7\%, $0.3 \%, 1.4 \%)$, iron only $(2.1 \%, 0.5 \%, 1.6 \%)$, iron and folic acid $(2.0 \%, 0.5 \%, 1.5 \%)$, folic acid only $(2.8 \%$, $0.5 \%, 2.3 \%)$, and none of the supplements $(2.2 \%$, $0.5 \%, 1.8 \%)$.

In the cohort, supplement user groups differed from one another across multiple health and socioeconomic characteristics (table 1). Compared with non-users, multivitamin users tended to be older, primiparous, and more educated, have higher family incomes, and were less likely to smoke and more likely to be born in Sweden. Folic acid users were similar to multivitamin users but were more likely than multivitamin users to be immigrants, to be smokers, and have epilepsy or be taking antiepileptics. Conversely, mothers taking iron only, or in combination with folic acid, tended to be younger, multiparous, and less educated, and to have lower family incomes, and were more likely to be immigrants and smokers than non-users. Depression and antidepressant use were slightly more prevalent among all categories of supplement users versus nonusers.

\section{Primary analyses \\ Multivitamin}

Regression analysis indicated that multivitamin use was associated with a lower likelihood of ASD with intellectual disability compared with non-use of multivitamins, iron, and folic acid in all analyses (odds ratio 0.69, 95\% confidence interval 0.57 to $0.84)$. Point estimates from the sibling control $(0.77$, 0.52 to 1.15$)$ and propensity score analyses ( 0.68 , 0.54 to 0.86 ) were similar, although the sibling control estimate had a wider confidence interval that included 1.0 and was therefore not statistically significant (table 2). Multivitamin use was not associated with ASD without intellectual disability in any analysis.

\section{Iron and folic acid}

Although imprecision owing to small numbers of folic acid only users was evident, there was a suggestion of an increased association between folic acid use and ASD without intellectual disability in the multivariable regression (odds ratio 1.29, 95\% confidence interval 0.99 to 1.67$)$ and sibling control (1.70, 0.94 to 3.10) analyses, that was attenuated in the propensity score analysis (1.10, 0.83 to 1.48). Iron use was not associated with ASD with or without intellectual disability in any analysis. 


\begin{tabular}{|c|c|c|c|c|c|}
\hline \multirow[b]{2}{*}{ Characteristics } & \multicolumn{5}{|l|}{ Supplement } \\
\hline & $\begin{array}{l}\text { Multivitamins } \\
(\mathrm{n}=62840)\end{array}$ & $\begin{array}{l}\text { Iron only } \\
(\mathrm{n}=90138)\end{array}$ & $\begin{array}{l}\text { Iron and folic } \\
\text { acid }(n=25445)\end{array}$ & $\begin{array}{l}\text { Folic acid only } \\
(n=2789)\end{array}$ & None $(n=91895)$ \\
\hline ASD & $1064(1.7)$ & $1872(2.1)$ & $516(2.0)$ & $78(2.8)$ & $2045(2.2)$ \\
\hline \multicolumn{6}{|l|}{ ASD with intellectual disability: } \\
\hline Yes & $158(0.3)$ & $422(0.5)$ & $138(0.5)$ & $15(0.5)$ & $430(0.5)$ \\
\hline No & $906(1.4)$ & $1450(1.6)$ & $378(1.5)$ & $63(2.3)$ & $1615(1.8)$ \\
\hline Boys & $31990(50.9)$ & $45933(51.0)$ & $12855(50.5)$ & $1451(52.0)$ & $47683(51.9)$ \\
\hline \multicolumn{6}{|l|}{ Parity: } \\
\hline 1 & $32488(51.7)$ & $38780(43.0)$ & $10628(41.8)$ & $1335(47.9)$ & $41679(45.4)$ \\
\hline 2 & 21927 (34.9) & $33258(36.9)$ & $9161(36.0)$ & $995(35.7)$ & $33509(36.5)$ \\
\hline$\geq 3$ & $8425(13.4)$ & $18100(20.1)$ & $5656(22.2)$ & $459(16.5)$ & $16707(18.2)$ \\
\hline \multicolumn{6}{|l|}{ Maternal education: } \\
\hline$\geq 12$ years & $34633(55.1)$ & $36613(40.6)$ & $10281(40.4)$ & $1365(48.9)$ & $43019(46.8)$ \\
\hline Missing data & $242(0.4)$ & $611(0.7)$ & $258(1.0)$ & $17(0.6)$ & $499(0.5)$ \\
\hline Maternal country of origin, Sweden & $51571(82.1)$ & $62296(69.1)$ & $16399(64.5)$ & $2164(77.6)$ & $70698(76.9)$ \\
\hline Highest fifth of family income & $27051(43.1)$ & $25012(27.8)$ & $6978(27.4)$ & $1078(38.7)$ & $33122(36.0)$ \\
\hline Mean (SD) maternal age (years) & $31.3(4.7)$ & $30.2(5.2)$ & $30.5(5.3)$ & $31.4(5.0)$ & $30.9(5.0)$ \\
\hline \multicolumn{6}{|l|}{ Maternal smoking: } \\
\hline Yes & $3434(5.5)$ & $8229(9.1)$ & $2080(8.2)$ & $200(7.2)$ & $6523(7.1)$ \\
\hline No & $57080(90.8)$ & $78636(87.2)$ & $22393(88.0)$ & $2502(89.7)$ & $55576(60.5)$ \\
\hline Missing data & $2326(3.7)$ & $3273(3.6)$ & $972(3.8)$ & $87(3.1)$ & $29796(32.4)$ \\
\hline \multicolumn{6}{|l|}{ Maternal body mass index $\left(\mathrm{kg} / \mathrm{m}^{2}\right)$ : } \\
\hline Underweight (<18.5) & $1715(2.7)$ & $2435(2.7)$ & 729 (2.9) & $49(1.8)$ & $1503(1.6)$ \\
\hline Normal (18.5-24.9) & $40593(64.6)$ & $53299(59.1)$ & $14960(58.8)$ & $1655(59.3)$ & $36995(40.3)$ \\
\hline Overweight (25-29.9) & $10287(16.4)$ & $17637(19.6)$ & $5065(19.9)$ & $526(18.9)$ & $12675(13.8)$ \\
\hline Obese $(\geq 30)$ & $3207(5.1)$ & $6131(6.8)$ & $1884(7.4)$ & $205(7.4)$ & $4991(5.4)$ \\
\hline Missing data & $7038(11.2)$ & $10636(11.8)$ & $2807(11.0)$ & $354(12.7)$ & $35731(38.9)$ \\
\hline \multicolumn{6}{|l|}{ Maternal medication use: } \\
\hline Antidepressant & $1017(1.6)$ & $1368(1.5)$ & $367(1.4)$ & $38(1.4)$ & $916(1.0)$ \\
\hline Antiepileptic & $118(0.2)$ & $119(0.1)$ & $215(0.8)$ & $65(2.3)$ & $125(0.1)$ \\
\hline \multicolumn{6}{|l|}{ Maternal neuropsychiatric conditions: } \\
\hline Anxiety disorders & $870(1.4)$ & 1209 (1.3) & $347(1.4)$ & $35(1.3)$ & $1176(1.3)$ \\
\hline Autism & $64(0.1)$ & $127(0.1)$ & $34(0.1)$ & $5(0.2)$ & $120(0.1)$ \\
\hline Bipolar disorder & $115(0.2)$ & $159(0.2)$ & $56(0.2)$ & $9(0.3)$ & $176(0.2)$ \\
\hline Depression & $2036(3.2)$ & $2571(2.9)$ & $695(2.7)$ & $82(2.9)$ & $2434(2.7)$ \\
\hline Epilepsy & $347(0.6)$ & $391(0.4)$ & $282(1.1)$ & $69(2.5)$ & $470(0.5)$ \\
\hline Intellectual disability & $19(0.0)$ & $68(0.1)$ & $20(0.1)$ & $2(0.1)$ & $67(0.1)$ \\
\hline Non-affective psychotic disorders & $140(0.2)$ & $258(0.3)$ & $83(0.3)$ & $11(0.4)$ & $281(0.3)$ \\
\hline Stress disorders & $1210(1.9)$ & $1668(1.9)$ & $475(1.9)$ & $70(2.5)$ & $1648(1.8)$ \\
\hline
\end{tabular}

\section{Sensitivity analyses}

Multivitamin categorization

Wetested whether theassociation between multivitamin use and ASD with intellectual disability was sensitive to alternate categorization and analytic approaches. Analysis of women who used multivitamins without additional iron or folic acid yielded an odds ratio estimate of 0.60 (95\% confidence interval 0.44 to 0.84 ); also see eTable 4 in web appendix 1). In analyses using indicator variables for supplement use and adjusted for the other supplements, estimates for multivitamins and ASD with intellectual disability in the regression (odds ratio $0.71,95 \%$ confidence interval 0.59 to 0.84 ) and sibling analyses $(0.80,0.55$ to 1.17$)$ were similar to estimates from primary analyses (see eTable 5 in web appendix 1).

Maternal hospital stays

Evidence of an increased risk of child ASD with folic acid use was limited to mothers with at least one hospital stay during pregnancy (odds ratio 1.59,
95\% confidence interval 1.21 to 2.10 for any ASD; see eTable 6 in web appendix 1). No evidence of an increased risk with folic acid use was seen in mothers with no hospital stays (0.84, 0.54 to 1.30 for any ASD). Importantly, the associations of multivitamin use with lower risk of ASD with intellectual disability persisted in this sensitivity analysis (no hospital stays: $0.64,0.47$ to 0.87 ; any hospital stays: 0.75 , 0.58 to 0.97$)$.

\section{Unobserved confounding}

We found evidence to suggest that the association between multivitamin use and ASD with intellectual disability was not likely to be wholly a result of unobserved confounding. For example, if a hypothetical binary confounder reduced the risk of ASD with intellectual disability by $50 \%$ and was twice as prevalent in multivitamin users $(40 \%)$ versus nonusers $(20 \%)$, the propensity score odds ratio estimate of 0.69 (95\% confidence interval 0.57 to 0.84 ) would attenuate to 0.78 (0.64 to 0.95 ). 


\begin{tabular}{|c|c|c|c|c|c|}
\hline \multirow[b]{2}{*}{ Sample } & \multicolumn{2}{|l|}{ Sibling CLR* model } & \multirow{2}{*}{$\begin{array}{l}\text { Propensity score } \\
\text { OLR and GEE modelst } \\
\text { Matched }\end{array}$} & \multicolumn{2}{|c|}{ Multivariable GEE $¥$ model } \\
\hline & Unadjusted & Adjusted§ & & Unadjusted & Adjusted \\
\hline \multicolumn{6}{|c|}{ ASD with intellectual disability: } \\
\hline Multivitamin & $0.60(0.42$ to 0.86$)$ & $0.77(0.52$ to 1.15$)$ & $0.68(0.54$ to 0.86$)$ & $0.54(0.45$ to 0.65$)$ & $0.69(0.57$ to 0.84$)$ \\
\hline Iron & 0.84 (0.64 to 1.10$)$ & $0.90(0.67$ to 1.21$)$ & $0.96(0.82$ to 1.13$)$ & $0.99(0.87$ to 1.14$)$ & $0.95(0.83$ to 1.11$)$ \\
\hline Iron and folic acid & $0.81(0.54$ to 1.22$)$ & $0.99(0.63$ to 1.57$)$ & $1.06(0.86$ to 1.30$)$ & $1.14(0.93$ to 1.38$)$ & $1.03(0.84$ to 1.26$)$ \\
\hline Folic acid & $0.81(0.28$ to 2.36$)$ & $0.94(0.29$ to 3.04$)$ & $1.14(0.64$ to 2.04$)$ & $1.16(0.69$ to 1.94$)$ & $1.20(0.71$ to 2.01$)$ \\
\hline \multicolumn{6}{|c|}{ ASD without intellectual disability: } \\
\hline Multivitamin & $0.93(0.79$ to 1.10$)$ & $1.00(0.83$ to 1.20$)$ & $0.95(0.85$ to 1.06$)$ & $0.82(0.75$ to 0.89$)$ & $0.94(0.85$ to 1.03$)$ \\
\hline Iron & $0.93(0.81$ to 1.07$)$ & $0.96(0.81$ to 1.12$)$ & $0.95(0.88$ to 1.04$)$ & $0.92(0.85$ to 0.98$)$ & $0.96(0.89$ to 1.04$)$ \\
\hline Iron and folic acid & 0.91 (0.73 to 1.13$)$ & $1.00(0.78$ to 1.29$)$ & $0.84(0.74$ to 0.94$)$ & $0.85(0.76$ to 0.95$)$ & $0.89(0.79$ to 1.00$)$ \\
\hline Folic acid & $1.46(0.87$ to 2.45$)$ & $1.70(0.94$ to 3.10$)$ & $1.10(0.83$ to 1.48$)$ & $1.30(1.00$ to 1.68$)$ & $1.29(0.99$ to 1.67$)$ \\
\hline \multicolumn{6}{|l|}{ Any ASD: } \\
\hline Multivitamin & $0.86(0.74$ to 1.00$)$ & $0.95(0.81$ to 1.13$)$ & $0.86(0.78$ to 0.95$)$ & 0.76 (0.71 to 0.82$)$ & 0.89 (0.82 to 0.97$)$ \\
\hline Iron & 0.91 (0.80 to 1.03$)$ & 0.95 (0.83 to 1.09) & $0.93(0.87$ to 1.01$)$ & 0.93 (0.87 to 0.99) & $0.96(0.90$ to 1.03$)$ \\
\hline Iron and folic acid & 0.89 (0.73 to 1.07$)$ & 1.01 (0.81 to 1.25$)$ & 0.89 (0.81 to 0.99) & $0.91(0.82$ to 1.00$)$ & $0.92(0.83$ to 1.02$)$ \\
\hline Folic acid & $1.30(0.82$ to 2.06$)$ & $1.48(0.87$ to 2.51$)$ & $1.17(0.89$ to 1.51$)$ & 1.27 (1.01 to 1.60$)$ & 1.27 (1.01 to 1.60$)$ \\
\hline
\end{tabular}

$\mathrm{CLR}=$ conditional logistic regression; $\mathrm{OLR}=$ ordinary logistic regression; $\mathrm{GEE}=$ generalised estimating equation logistic regression.

Analytic sample sizes are documented in supplemental eTable 1, ranging from 15575 mothers and 16086 children (folic acid use and ASD with intellectual disability propensity score analysis) to 178083 mothers and 273107 children (any ASD multivariable sample analyses).

* Matched on birth mother.

tPropensity scores were calculated with covariates (see 9 below) as predictors of supplement use in ordinary logistic regression models (maternal age was a continuous covariate); in the matched sample, propensity scores were used as predictors of ASD in generalised estimating equation logistic regression models grouped by birth mother.

‡Grouped by birth mother.

§Adjusted for child characteristics (sex and birth year) and parity.

IAdjusted for child characteristics (sex, birth year, and years resided in Stockholm County), socioeconomic indicators (education, family income, and maternal birth country), maternal

characteristics (age, body mass index, parity, smoking status), medication use during pregnancy (antidepressants or antiepileptics), and maternal neuropsychiatric conditions (anxiety

disorders, autism, bipolar disorder, depression, epilepsy, intellectual disability, non-affective psychotic disorders, and stress disorders).

\section{Discussion}

The present population based study used sibling controls, propensity score matching, and multivariable regression to examine associations of maternal nutritional supplementation during pregnancy and risk of autism spectrum disorder (ASD) in offspring. We could not rule out the possibility that multivitamin supplementation may be inversely associated with ASD with intellectual disability. Evidence that either iron or folic acid use were inversely associated with ASD was inconsistent.

\section{Strengths and weaknesses of this study}

The main strength of this study is the relatively large population based sample in which advanced analytic methods were used to gauge robustness of findings. In addition, the case finding approach covered all pathways to ASD care and services in Stockholm County, Sweden, in the context of a universal healthcare system, thus increasing the likelihood that cases of ASD were identified.

Sibling control ${ }^{32}$ and propensity score matching ${ }^{33}$ analyses were used to examine whether results obtained using conventional multivariable regression were likely to be influenced by either observed or unobserved confounding. Such confounding is likely, given that nutritional supplement users were different from non-users in ways that may influence ASD risk. Propensity score matching was effective in balancing covariate distributions between exposed and unexposed children (see efig 1 in web appendix 1 ). Multivariable regression and propensity score findings were similar and suggest that observed confounding is not responsible for observed associations, though unobserved confounding remains a potential concern in these two analyses.

By comparing within sibling matched sets, sibling analyses neutralize potential shared observed and unobserved confounders that might predict both supplement use and ASD in childhood. Sibling studies have lower sample sizes, which lead to imprecise estimates and high type II error, as well as other limitations. ${ }^{34}$ In particular, sibling analyses have greater susceptibility to potential non-shared confounders that change between subsequent pregnancies (eg, nutritional depletion in the case of short intervals between pregnancies) and random measurement error of the exposure, which would be expected to attenuate associations compared with conventional estimates. ${ }^{34}$ Since the sibling estimate was not statistically significant at $\alpha=0.05$, it warrants cautious interpretation. In the present study, the sibling analysis point estimate was similar to the multivariable regression estimate, supporting the possibility that unobserved confounding owing to shared characteristics was not driving this association. Together, the three analyses appeared to point toward a potential inverse association between multivitamin use and ASD with intellectual disability.

The study has substantial limitations, such as the potential for confounding. Our calculations of the potential impact of unmeasured confounding suggest that to nullify the potential association between multivitamin use and ASD with intellectual disability 
an unmeasured confounder would have to be a strong protective factor against ASD with intellectual disability, exerting an effect above and beyond the covariates matched in propensity score analyses (see eTable 7 in web appendix 1). An editor suggested that a maternal trait that predicts multivitamin usehigher intelligence-may decrease the risk of ASD with intellectual disability. However, analyses controlled for maternal education level, intellectual disability, and ASD. Moreover, the sibling control analyses, which would be expected to control for such a confounder, produced point estimates not inconsistent with the regression analysis. Behavioural confounding is also another possibility, as healthy lifestyle is a well known confounder in studies of nutritional supplement use. ${ }^{18}$ Our analyses controlled for maternal body mass index but were unable to assess the contribution of diet or other nutritional supplements. Existing research on antenatal diet and ASD is limited and further study is recommended. ${ }^{27}$ In addition, the associations between multivitamin use and ASD with intellectual disability could be related to ASD, intellectual disability, or both. This could not be examined in the present study.

The observation that folic acid use was associated with higher risk only in women admitted to hospital during pregnancy and the absence of associations between the iron and folic acid group and ASD suggests confounding by causes of hospital stays such as chronic somatic or psychiatric conditions, acute illness, pregnancy related disorders, or psychosocial distress. ${ }^{35-37}$ Whether this occurs through structural pathways (eg, folic acid being prescribed at higher rates among women staying in hospital during pregnancy) or through biological pathways (eg, folate insufficiency as a result of a chronic condition or medication) is unknown. Potential exposure to antifolates through either maternal history of epilepsy ${ }^{38}$ or use of an antiepileptic medication ${ }^{39}$ during pregnancy have both been associated with increased risk of ASD in children and were more prevalent in the folic acid group, making confounding by indication possible.

Another potential limitation was categorization of the multivitamin group as having used multivitamins with or without additional iron or folic acid, and the use of non-overlapping supplement groups. However, sensitivity analyses examining women who only used multivitamins without iron and folic acid and analyses using indicator variables for supplement use instead of supplement groups yielded similar estimates to those from primary analyses.

Assessment of supplement use was limited, since type, timing, and dose of supplements could not be determined. Changes to nutritional status or supplement use after the first antenatal visit could not be assessed. It is possible that the reported supplement was not taken, or a supplement was taken but not reported. While the extent of exposure misclassification is unknown, prevalences of supplement use observed here are in the range of estimates from other studies in the Swedish population during the same period. ${ }^{4041}$
Even if a causal association could be established between multivitamin use and ASD with intellectual disability, it cannot be determined from the data whether there is a critical window for exposure, what specific nutrients or combinations thereof may be responsible, or what dose is required. External validity is also not assured, since different populations have different backgrounds of supplement use, fortification, dietary patterns, and health risks. Lastly, although the study featured a relatively large sample size, small cell counts owing to rare outcomes made it difficult to obtain precise estimates from lower powered analyses, particularly the sibling control analyses.

\section{Comparison with other studies}

Our findings agree with some of the existing literature. The three case-control studies reporting reduced risk of ASD with antenatal vitamins, ${ }^{14}$ iron, ${ }^{13}$ and folic acid ${ }^{42}$ were from the CHildhood Autism Risks from Genetics and Environment (CHARGE) study in the USA.

Interestingly, lower mean folic acid intake in CHARGE was found for children with ASD with a lower cognitive function but not for ASD with higher functioning, suggesting that nutrients may differentially affect ASD risk depending on co-occurrence with intellectual disability. In the prospective Norwegian Mother and Child Cohort study, maternal folic acid intake, but not other vitamins and minerals, in the periconceptional period and early pregnancy, was associated with lower risk of ASD. ${ }^{11}$ The Danish National Birth Cohort study reported null associations between folic acid and multivitamin intake and ASD or any of its subtypes. ${ }^{12}$ To date, all published studies of maternal folate biomarkers and ASD or autistic traits in children have found null relations. ${ }^{43}{ }^{44}$ However, these biomarker studies also evaluated nutrient intake and reported associations with reduced ASD symptoms.

Timing of exposure could have played a role in differences among findings for iron and folic acid. In the current study, supplementation was assessed at a median of 10.7 weeks' pregnancy (interquartile range 9.0-12.7 weeks). The observed association for folic acid intake in CHARGE was for use through the first month of pregnancy, and findings were null after the second month of pregnancy. For the Norwegian Mother and Child Cohort study, the protective association pertained to use through the first trimester, whereas the Danish National Birth Cohort study examined use through eight weeks' gestation. Conversely, biomarker studies with positive findings had assessed maternal folate after the first trimester.

Another possibility that may contribute to differences between studies is that the background nutritional contexts of the countries in which the studies are conducted differ. For example, while the USA implements folic acid fortification, Sweden, Norway, and Denmark do not. Fish oil supplements are routinely taken in Norway by nearly $60 \%$ of pregnant women ${ }^{45}$ but are rarely used in Sweden. Similarly, serum 25-hydroxyvitamin D levels in pregnant 
women are lower in Sweden ${ }^{46}$ than in Denmark, ${ }^{47}$ corresponding with the difference in latitude.

Such differences may help to explain why a reduced risk of ASD with iron or folic acid use was not observed. Data suggest that nutritional intake may differ between cohorts. ${ }^{48}$ Participants in the Norwegian Mother and Child Cohort study reported combined daily dietary and supplementary iron ( $40.4 \mathrm{mg}$ ) and folic acid (615 $\mu \mathrm{g})^{45}$ concentrations similar to levels associated with protection against ASD in the CHARGE study (57.1 mg iron ${ }^{13}$ and $600 \mu \mathrm{g}$ folic acid ${ }^{42}$ ). Participants in the Danish National Birth Cohort study reported $575 \mu \mathrm{g}$ combined dietary and supplementary folic acid. ${ }^{49}$ In contrast, combined dietary and supplementary iron (15.0 mg) and folic acid (430 $\mu \mathrm{g})$ intake among Swedish pregnant women ${ }^{48}$ were less than in the CHARGE study or the Norwegian Mother and Child Cohort study. Taken together, it is not unreasonable to conclude that effects of supplements taken in one country may differ from those in another country, depending on whether combined dietary and supplementary levels of nutrients reach a threshold necessary to elicit protection.

\section{Conclusions and policy implications}

We observed a potential inverse association between maternal multivitamin supplement use and ASD with intellectual disability in offspring. Whether the association is specific to autism or reflects the risk of intellectual disability needs to be explored in future research. Given the current understanding and strength of evidence supporting the importance of nutritional supplementation during pregnancy, these results on their own should not change current practice. Still, these findings raise questions that warrant investigation. A sufficient body of mechanistic evidence supporting the finding does not yet exist. Verification in independent epidemiologic investigations with improved exposure assessment, perhaps in randomised studies, is necessary before recommending a change to current practice.

Contributors: $\mathrm{CM}$ and $\mathrm{CD}$ provided the data used in the study. All authors designed the study. EAD performed the data analysis and drafted the manuscript. All authors had full access to all of the data (including statistical reports and tables) in the study and can take responsibility for the integrity of the data and the accuracy of the data analysis. All authors interpreted data, critically revised, and approved the final manuscript.

Funding: This study was funded by the National Institutes of Health (1 R21 ES023760-01A1, "Early life vitamin D levels and risk of autism spectrum disorders") Swedish Research Council, National Institute for Health Research Biomedical Research Centre Bristol. Funders facilitated EAD and BKL to perform their authorship responsibilities. Researchers were independent from funders.

Competing interests: All authors have completed the ICMJE uniform disclosure form at www.icmje.org/coi_disclosure.pdf and declare: no support from any organisation for the submitted work; no financial relationships with any organisations that might have an interest in the submitted work in the previous three years; no other relationships or activities that could appear to have influenced the submitted work.

Ethical approval: This research has ethical approval as part of Psychiatry Sweden "Psykisk ohälsa, psykiatrisk sjukdom: förekomst och etiologi," [Mental health and psychiatric disorders-prevalence and aetiology] approved by the Stockholm regional ethical review board (No 2010/1185-31/5).
Data sharing: The statistical code is available from the corresponding author.

Transparency: The lead author (EAD) affirms that the manuscript is an honest, accurate, and transparent account of the study being reported; that no important aspects of the study have been omitted; and that any discrepancies from the study as planned have been explained.

This is an Open Access article distributed in accordance with the Creative Commons Attribution Non Commercial (CC BY-NC 4.0) license, which permits others to distribute, remix, adapt, build upon this work non-commercially, and license their derivative works on different terms, provided the original work is properly cited and the use is noncommercial. See: http://creativecommons.org/licenses/by-nc/4.0/

1 Gaugler T, Klei L, Sanders SI, et al. Most genetic risk for autism resides with common variation[published Online First: 2014 Jul 20]. Nat Genet 2014;46:881-5. doi:10.1038/ng.3039

2 Sandin S, Lichtenstein P, Kuja-Halkola R, Larsson H, Hultman CM, Reichenberg A. The familial risk of autism[published Online First: 2015 Apr 1]. JAMA 2014;311:1770-7. doi:10.1001/ jama.2014.4144

3 Lyall K, Schmidt RJ, Hertz-Picciotto I. Maternal lifestyle and environmental risk factors for autism spectrum disorders. Int J Epidemiol 2014:43:443-64. doi:10.1093/ije/dyt282

4 Newschaffer CJ, Croen LA, Daniels J, et al. The epidemiology of autism spectrum disorders[published Online First: 2007 Mar 21]. Annu Rev Public Health 2007;28:235-58. doi:10.1146/annurev. publhealth.28.021406.144007

5 Stoner R, Chow ML, Boyle MP, et al. Patches of disorganization in the neocortex of children with autism[published Online First: 2014 Mar 27]. N Engl J Med 2014;370:1209-19. doi:10.1056/ NEJMoa1307491

6 Bale TL. Epigenetic and transgenerational reprogramming of brain development[published Online First: 2015 Apr 29]. Nat Rev Neurosci 2015;16:332-44. doi:10.1038/nrn3818

7 Brown AS, Susser ES. Prenatal nutritional deficiency and risk of adult schizophrenia. Schizophr Bull 2008;34:1054-63. doi:10.1093/ schbul/sbn096

8 Xu MQ, Sun WS, Liu BX, et al. Prenatal malnutrition and adult schizophrenia: further evidence from the 1959-1961 Chinese famine[published Online First: 2009 Jan 20]. Schizophr Bull 2009:35:568-76. doi:10.1093/schbul/sbn168

9 DeVilbiss EA, Gardner RM, Newschaffer CJ, Lee BK. Maternal folate status as a risk factor for autism spectrum disorders: a review of existing evidence[published Online First: 2015 Aug 5]. BrJ Nutr 2015;114:663-72. doi:10.1017/S0007114515002470

10 Schmidt RJ, Tancredi DJ, Ozonoff S, et al. Maternal periconceptional folic acid intake and risk of autism spectrum disorders and developmental delay in the CHARGE (CHildhood Autism Risks from Genetics and Environment) case-control study. Am J Clin Nutr 2012;96:80-9. doi:10.3945/ajcn.110.004416

11 Surén P, Roth C, Bresnahan M, et al. Association between maternal use of folic acid supplements and risk of autism spectrum disorders in children. JAMA 2013;309:570-7. doi:10.1001/ jama.2012.155925

12 Virk J, Liew Z, Olsen J, Nohr EA, Catov JM, Ritz B. Preconceptional and prenatal supplementary folic acid and multivitamin intake and autism spectrum disorders. Autism 2016;20:710-8. doi:10.1177/1362361315604076

13 Schmidt RJ, Tancredi DJ, Krakowiak P, Hansen RL, Ozonoff S. Maternal intake of supplemental iron and risk of autism spectrum disorder. $A m$ J Epidemiol 2014:180:890-900. doi:10.1093/aje/kwu208

14 Schmidt RJ, Hansen RL, Hartiala J, et al. Prenatal vitamins, one-carbon metabolism gene variants, and risk for autism[published Online First: 2011 Jul]. Epidemiology 2011;22:476-85. doi:10.1097/ EDE.0b013e31821d0e30

15 Magnusson C, Rai D, Goodman A, et al. Migration and autism spectrum disorder: population-based study. Br J Psychiatry 2012;201:109-15 doi:10.1192/bjp.bp.111.095125

16 Rai D, Lee BK, Dalman C, Golding J, Lewis G, Magnusson C. Parental depression, maternal antidepressant use during pregnancy, and risk of autism spectrum disorders: population based case-control study[published Online First: 2013 Apr 19]. BMJ 2013;346:f2059. doi:10.1136/bmj.f2059

17 Szatmari P, White J, Merikangas KR. The use of genetic epidemiology to guide classification in child and adult psychopathology[published Online First: 2007 Oct]. Int Rev Psychiatry 2007;19:483-96. doi:10.1080/09540260701563619

18 Lawlor DA, Davey Smith G, Kundu D, Bruckdorfer KR, Ebrahim S. Those confounded vitamins: what can we learn from the differences between observational versus randomised trial evidence?[published Online First: 2004 May 22]. Lancet 2004;363:1724-7. doi:10.1016/ S0140-6736(04)16260-0 
19 Idring S, Rai D, Dal H, et al. Autism spectrum disorders in the Stockholm Youth Cohort: design, prevalence and validity. PLoS One 2012;7:e41280. doi:10.1371/journal.pone.0041280

20 The Swedish Medical Birth Register - A summary of content and quality, The National Board of Health and Welfare: Centre for Epidemiology [Internet]. 2003. Available from: http://www. socialstyrelsen.se/publikationer2003/2003-112-3

21 Axén M. ADHD, lindrig utvecklingsstörning och autismspektrumtillstånd hos barn, ungdomar och vuxna [ADHD, mild mental retardation and autism spectrum disorders in children, adolescents and adults]. Stockholm: Stockholms läns landsting 2010

22 Cawley S, Mullaney L, McKeating A, Farren M, McCartney D, Turner MJ. A review of European guidelines on periconceptional folic acid supplementation[published Online First: 2015 Sep 10]. Eur J Clin Nutr 2016;70:143-54. doi:10.1038/ejcn.2015.131

23 SFOG. Halso-overvakning vid normal graviditet (Routine antenata care). ARG rapport 1991;21:37-38.

24 Källén B. Use of folic acid supplementation and risk for dizygotic twinning[published Online First: 2004 Nov]. Early Hum Dev 2004;80:143-51. doi:10.1016/j.earlhumdev.2004.06.002

25 George L, Mills IL, Johansson ALV, et al. Plasma folate levels and risk of spontaneous abortion[published Online First: 2002 Oct 16]. JAMA 2002;288:1867-73. doi:10.1001/jama.288.15.1867

26 Cnattingius S, Ericson A, Gunnarskog J, Källén B. A quality study of a medical birth registry. Scand / Soc Med 1990;18:143-8. doi:10.1177/140349489001800209

27 Lyall K, Croen L, Daniels J, et al. The Changing Epidemiology of Autism Spectrum Disorders[published Online First: 2017 Jan 10]. Annu Rev Public Health 2017;38:81-102. doi:10.1146/annurevpublhealth-031816-044318

28 Rai D, Lewis G, Lundberg M, et al. Parental socioeconomic status and risk of offspring autism spectrum disorders in a Swedish populationbased study. J Am Acad Child Adolesc Psychiatry 2012;51:467-476.e6.

29 Sullivan PF, Magnusson C, Reichenberg A, et al. Family history of schizophrenia and bipolar disorder as risk factors for autism[published Online First: 2012 Jul 04]. Arch Gen Psychiatry 2012;69:1099-103. doi:10.1001/ archgenpsychiatry.2012.730

30 Stephansson O, Granath F, Svensson T, Haglund B, Ekbom A, Kieler H. Drug use during pregnancy in Sweden - assessed by the Prescribed Drug Register and the Medical Birth Register[published Online First 2011 Mar 10]. Clin Epidemiol 2011;3:43-50. doi:10.2147/CLEP. S16305

31 Lin DY, Psaty BM, Kronmal RA. Assessing the sensitivity of regression results to unmeasured confounders in observational studies[published Online First: 1998 Sep]. Biometrics 1998;54: 948-63. doi:10.2307/2533848

32 Susser E, Eide MG, Begg M. Invited commentary: The use of sibship studies to detect familial confounding[published Online First: 2010 Jul 14]. Am J Epidemiol 2010;172:537-9. doi:10.1093/aje/ kwq196

33 Stuart EA. Matching methods for causal inference: A review and a look forward[published Online First: 2010 Feb 1]. Stat Sci 2010;25:1-21. doi:10.1214/09-STS313

34 Frisell T, Öberg S, Kuja-Halkola R, Sjölander A. Sibling comparison designs: bias from non-shared confounders and measurement error[published Online First: 2012 Sep]. Epidemiology 2012;23: 713-20. doi:10.1097/EDE.0b013e31825fa230

35 Rostad B, Schei B. Factors predicting antenatal hospital admission in pregnancy. Scand J Prim Health Care 1998;16:85-9. doi:10.1080/028134398750003223
36 Lindholm ES, Altman D, Norman M, Blomberg M. Health Care Consumption during Pregnancy in relation to Maternal Body Mass Index: A Swedish Population Based Observational Study. J Obes 2015;2015:215683.

37 Lee BK, Magnusson C, Gardner RM, et al. Maternal hospitalization with infection during pregnancy and risk of autism spectrum disorders[published Online First: 2014 Sep 16]. Brain Behav Immun 2015;44:100-5. doi:10.1016/j.bbi.2014.09.001

38 Leonard H, de Klerk N, Bourke J, Bower C. Maternal health in pregnancy and intellectual disability in the offspring a population-based study[published Online First: 2005 Sep 22]. Ann Epidemiol 2006;16:448-54. doi:10.1016/j. annepidem.2005.05.002

39 Christensen J, Grønborg TK, Sørensen MJ, et al. Prenatal valproate exposure and risk of autism spectrum disorders and childhood autism[published Online First: 2013 Apr 24]. JAMA 2013;309: 1696-703. doi:10.1001/jama.2013.2270

40 Granfors M, Augustin H, Ludvigsson J, Brekke HK. No association between use of multivitamin supplement containing vitamin D during pregnancy and risk of Type 1 Diabetes in the child[published Online First: 2015 Nov 11]. Pediatr Diabetes 2016;17:525-30. doi:10.1111/pedi.12334

41 Ericson A, Källén B, Åberg A. Use of multivitamins and folic acid in early pregnancy and multiple births in Sweden. Twin Res 2001;4: 63-6. doi:10.1375/1369052012155

42 Schmidt RI, Tancredi DJ, OzonoffS, et al. Maternal periconceptional folic acid intake and risk of autism spectrum disorders and developmental delay in the ASD (CHildhood Autism Risks from Genetics and Environment) case-control study. Am J Clin Nutr 2012;96:80-9. doi:10.3945/ajen.110.004416

43 Braun JM, Froehlich T, Kalkbrenner A, et al. Brief report: are autistic-behaviors in children related to prenatal vitamin use and maternal whole blood folate concentrations?] Autism Dev Disord 2014;44:2602-7. doi:10.1007/s10803-014-2114-x

44 Steenweg-de Graaff J, Ghassabian A, Jaddoe VWV, Tiemeier H, Roza SJ. Folate concentrations during pregnancy and autistic traits in the offspring. The Generation R Study[published Online First: 2014 Jul 31]. Eur J Public Health 2015;25:431-3. doi:10.1093/eurpub/ cku126

45 Haugen M, Brantsaeter AL, Alexander J, Meltzer HM. Dietary supplements contribute substantially to the total nutrient intake in pregnant Norwegian women[published Online First: 2008 Jul 22]. Ann Nutr Metab 2008:52:272-80 doi:10.1159/000146274

46 Brembeck P, Winkvist A, Olausson H. Determinants of vitamin D status in pregnant fair-skinned women in Sweden[published Online First: 2013 Feb 6]. Br J Nutr 2013;110:856-64. doi:10.1017/ S0007114512005855

47 Petersen SB, Olsen SF, Mølgaard C, et al. Maternal vitamin D status and offspring bone fractures: prospective study over two decades in Aarhus City, Denmark[published Online First: 2014 Dec 4]. PLoS One 2014;9:e114334. doi:10.1371/journal.pone.0114334

48 Lundqvist A, Johansson I, Wennberg A, et al. Reported dietary intake in early pregnant compared to non-pregnant women - a crosssectional study[published Online First: 2014 Nov 1]. BMC Pregnancy Childbirth 2014;14:373. doi:10.1186/s12884-014-0373-3

49 Mikkelsen TB, Osler M, Olsen SF. Validity of protein, retinol, folic acid and n-3 fatty acid intakes estimated from the food-frequency questionnaire used in the Danish National Birth Cohort[published Online First: 2006 Sep]. Public Health Nutr 2006;9:771-8. doi:10.1079/PHN2005883

Appendix: Supplementary materials 